\title{
Some Remarks on CSE Education in Germany
}

\author{
Hans-Joachim Bungartz \\ IPVS, Universität Stuttgart, Universitätsstraße 38, D-70569 Stuttgart, Germany \\ bungartz@ipvs.uni-stuttgart.de
}

\begin{abstract}
The way Computational Science and Engineering is taught today in Germany, often, seems to depend more on local boundary conditions than on the real needs. Actually, some very fundamental questions are still open or are, at least, answered in very different and not always satisfying ways. Among these are the issues of the right focus of such a program or of the appropriate balance between methodology and real applications, as well as the question whether to implement both undergraduate and graduate programs or a graduate one, only. In this contribution, we want to discuss these and other related topics, considering selected existing CSE study programs in German-speaking countries.
\end{abstract}

\section{Introduction}

The last decade has seen the birth of a lot of new study programs in Germany dealing with Computational Science and Engineering (CSE), Scientific Computing, or any kind of Computational Something, where Something stands for an established discipline from science or engineering such as physics or mechanics, e.g. This welcome development reflects the grown importance of computer-aided methods in general and numerical simulation or high-performance computing in particular. As a matter of fact, at the moment, the implementation of all kind of new programs is further supported by a suddenly rather dynamic situation concerning study programs, which is mainly due to the forthcoming or ongoing, more or less radical changes the Bologna agreement entails.

However, although these CSE-related programs all have similar strategic objectives, we can observe a variety of appearances - with differences that are sometimes far from being just minor ones. First, in Germany, CSE is not an established discipline in the sense that there exist schools or faculties of this name where the respective programs are housed naturally. Here, we sometimes really get jealous of these "Schools of Computing" or "Departments of Computational Sciences" existing elsewhere! As a consequence, the rootedness of a CSE program in an existing faculty and its (and that's the point) focus as regards content, in most cases just depend on those of its initiator(s). Hence, on the one hand, we have more application-driven programs like Computational Mechanics (COME) in München [3] or Computational Mechanics of Materials and Structures (COMMAS) 7] and Computational Physics [8] in Stuttgart, and, on the other hand, there are more methodology-dominated programs such as Scientific Computing in Berlin [2 or Computational Science and Engineering (CSE) in München [4]. 
A second issue is how to organize the CSE education - within an existing program or as a program of its own, and there either for graduate students only or for undergraduates, too. There are examples for all models: The faculties of mechanical engineering and civil engineering in Stuttgart, e.g., offer a study focus CSE [9] or Modelling and Simulation [10], resp., within their classical diploma programs. In contrast to that, the already mentioned COME, COMMAS, and or CSE as well as Computational Engineering (CE) 6] in Erlangen are all completely new programs, the former three being examples of pure master programs and the latter one consisting of both a bachelor and a master part.

Furthermore, we observe differences with respect to the program language. While several programs such as the ETH's Rechnergestützte Wissenschaften [1] hold to the German language, most pure master programs have been implemented as so-called international, i.e. English-based programs, since they want to (and actually do) attract international students, primarily, whereas the Germanlanguage programs cover most of the education of students from the Germanspeaking countries, at present. This raises the question of the target groups a CSE program wants to address. Apart from their nationality, this is also a question of disciplines. If a CSE master is designed by a mechanical engineering faculty for candidates holding a bachelor's degree in mechanical engineering, the profile of the students will be by far more homogeneous than it is in the case of the CSE program in München already mentioned, where just a bachelor's degree in some field of science or engineering is required.

This article aims at discussing these questions against the background of the needs of a modern CSE education as well as at opposing pros and cons of the respective model chosen. Although there will be statements for or against this or that way, it is, however, clear that solutions are always subject to boundary conditions such as local faculties, academic staff, and legal restrictions. Hence, there is no optimal way to be depicted, but just a collection of ideas that will, hopefully, initiate some reflections and discussions. The remainder of this paper is organized as follows. In Sect. 2, we discuss the topic of how to design CSE courses or programs and, in particular, whether to put a more concept-based or a more application-driven focus. The following Sect. 3 deals with the question whether CSE can just be seen as some kind of a specialization in the sense of either a minor topic within existing programs or some master graduate program accessible to students with some more or less conventional (first) degree, or whether a sound CSE education implies a complete restructuring of existing programs. Another issue, which will be addressed in Sect. 4, is the program language. Afterwards, in Sect. 5, we turn to the crucial question of degrees that qualify to enter the programs, and we discuss the chances and problems of a broader target group. Finally, some concluding remarks will close the discussion.

\section{Simulation Technology or Grand Challenges?}

Looking at the development of CSE, there have always been two driving forces of progress: general algorithmic improvements on the one hand and real applications on the other hand. The former - think of fast solvers, mesh refinement approaches, or parallelization strategies - are based on concepts such as the 
multilevel paradigm and have turned out to be of great importance for real-life problems, but they, typically, neither stem from nor are restricted to a specific field of application. The primary motivation rests on notions such as speed of convergence, orders of accuracy, or speedups, e.g. In contrast to that, the other driving force is fed by the wish to solve important practical problems, to tackle the "grand challenges". The history of finite element methods provides a nice example for this duality. While engineers, especially from the field of civil or structural engineering, were eagerly looking for ways to compute more and more complicated buildings (for example, the roof of the Olympic stadium in München was one of the early constructions designed with the help of nonlinear computations - done in Stuttgart, by the way), numerical analysts were more interested in approximation spaces, error orders, or strategies of adaptive mesh refinement. Both streams were successful in their endeavours and allowed for significant steps forward, but the crucial break-throughs were only possible when applications and fundamentals or general concepts and concrete problem scenarios met.

This double-track development is reflected in many CSE-related programs in Germany. For example, consider the two master programs on computational mechanics mentioned above, COME 3 at the Technische Universität München and COMMAS [7] at the Universität Stuttgart. Both curricula do without any core courses offered by members of the math faculties; in both of them, we only find one or two courses contributed by computer science (in COME this formerly was, in COMMAS it presently is the author's job). When, during an evaluation board meeting, for instance, the question arises whether this is really enough in a program carrying the word "computational" on its banner, the standard answer is of the type "of course, we teach numerical methods, too" or "but in the exercises of my course, students have to program, too". And this argument is followed by a shrugging reference to upper credit bounds that limit each kind of elbow-room for additional topics. To avoid misunderstandings: both COME and COMMAS are excellent programs - as proven by personal experience and external evaluation - and there can be no doubt that those who are responsible for curricula and courses did and do an extraordinarily good job. Nevertheless, the question remains whether we really come up with the interdisciplinary character of CSE by practicing an "if there is any need for some subjects of discipline XYZ, I'll deal with that, too" credo, whether interdisciplinary or, better, transdisciplinary really just means that the existing group of teachers prefers to cover a broader spectrum of topics themselves instead of enlarging the group of contributing experts. Of course, if the answer is no, it is no better to do it the other way round, regarding Scientific Computing as something very similar to numerical analysis, as many mathematicians sometimes tend to think, or as being basically a synonym of supercomputing, as especially those guys sitting on the TOP 500 machines in the big computing centres sometimes argue.

The main risk of a too much application-oriented curriculum seems to be a deficit in simulation technology, i.e. in teaching general algorithmic concepts or paradigms. Numerical schemes - sometimes rather outdated, sometimes really up-to-date - are presented as the state-of-the-art, but neither derived nor justified. Note that I'm not speaking of extensive proofs where everyone (sometimes 
including the lecturer) gets lost. If at all, students learn about hierarchical bases or multilevel solvers, e.g., just as being efficient tools for their specific tasks, but, at the end, they still lack of a deeper understanding of the way those work and of their potential. They learn to program and to use the few lines of the conjugate gradient algorithm, they - perhaps - learn about its main properties, but they don't really learn what happens in detail. All their doing is just caught in the narrow environment of their concrete flow problem to solve, e.g. The same holds for computer science. In many application-driven CSE-related programs, "computational" just means that some programming course is included, which by far does not reflect the needs of modern CSE. As a consequence, students are often unable to identify, carry over, or generalize the underlying concepts. Again, consider the other way round: The big risk of a pure simulation-technology-driven education with predominant mathematical and informatical components is the classical ivory tower scenario where sophisticated algorithms are derived, proven, and applied to examples as realistic as flow in a cubic motor. Obviously, such an unbalanced approach would not look better at all.

A better alternative is the really transdisciplinary approach of $C E[6]$ in Erlangen and CSE [4] in München. In the latter case, six faculties jointly run this master program. There is a math column, provided by the math faculty, a computer science column, provided by the informatics faculty, a scientific computing column focusing on simulation technology, and an application column, provided by experts from the respective faculties, where a broad spectrum of lines of specialization such as computational mechanics, fluid mechanics, electrodynamics, physics, or chemistry can be chosen. Of course, at the end, there are no experts in Computational ... for one specific field, but there are students who have learned the fundamentals of a key technology and to apply the latter to some highly relevant field of application. Critics see a drawback of this model in its possible superficiality, the well-known "hearing a lot but understanding nothing in depth". Nevertheless, we need the balance of concepts and applications.

How important and fruitful a really transdisciplinary CSE education is, can be seen every year at the Ferienakademie [5], a joint venture of the Technische Universität München, the Universität Erlangen-Nürnberg, and the Universität Stuttgart. The Ferienakademie is a kind of summer school for the best and most motivated students, financed by industry from the two German states Bayern and Baden-Württemberg, by the participating universities themselves, and by their respective alumni organizations. During two weeks end of September, a dozen of seminar-type courses are held in Southern Tyrol, each with two professors from two of the organizing universities, one assistant, and about fifteen student participants who, essentially, carry the program via their presentations and discussions. The course topics cover the whole spectrum of modern technical universities. The course Numerical simulation: from models to visualizations co-organized twice by the author addresses students from basically all disciplines involved in CSE. Actually, in 2003, the participating students represented Mathematics, Technomathematics, Computer Science, Physics, Computational Physics, Chemistry, Computational Engineering, Electrical Engineering, Chemical Engineering, Software Engineering, and Computational Mechanics. 
Of course, it is not always easy to handle such a mixture: computer scientists don't have the physical background to follow all discussions on turbulence in detail, a chemical engineer wonders what he can profit from a mathematician's presentation who solves the Laplace equation in a tricky way ("I have to solve flow problems!"), and computer scientists are astonished when a mechanical engineer asks basic questions on cache memories and on the RISC microprocessor architecture. Nevertheless, those experiences of different scientific cultures, different ways of giving a talk, different educational backgrounds, and different ways of tackling a given problem are crucial for real progress.

Finally, the given faculty structure at German universities is, to some extent, outdated, since the partitioning of today's scientific world should be definitely different from the one of a century ago. Instead of combining all groups involved in CSE activities in a powerful unit (call it school or department or however you want), there are computational minorities in most of the faculties, and there are permanent misunderstandings or even battles between pure and applied mathematicians, between floating-point and e-something computer scientists, between supercomputing engineers and experimental or production technology ones, and so on. As a result, there are CSE nuclei everywhere (which is not yet a drawback, of course) with non-coordinated curriculum activities (which definitely is one). Available expertise is not used, just because no one knows about it or, perhaps even worse, since everyone is convinced of a "we can do it on our own". Nevertheless, joint activities bringing together all experts from different faculties is definitely the way a transdisciplinary field such as CSE should be taught.

\section{Basic Education or Specialization?}

But let us turn to the appropriate format of CSE education, starting with a few examples. In Stuttgart, students of Mechanical Engineering, a classical 9semester German diploma program, have to choose one specialization after their Vordiplom, i.e. after the second year. The alternatives are Product Management and Design, Production Technology, Microsystem and Precision Engineering, General Mechanical Engineering, and, recently, also Computational Science and Engineering [9]. Those choosing the latter get at least about 36 ECTS credits of a more or less directly CSE-related education, and they have to make two student research projects in this field. Students of the Civil Engineering diploma program in Stuttgart face a similar situation, since Modelling and Simulation [10] has been newly added to the three classical specializations Structural Engineering, Traffic Engineering, and Water Engineering. Here, an important part of the years 3-5 is dedicated to this CSE specialization. By the way, in the first case, almost all courses are provided by members of the mechanical engineering faculty; in the second case, this is true apart from a few electives imported from mathematics. Hence, these are two more examples of a more or less "in-house" solution without a real integration of expertise from other disciplines. In contrast to that, for the Computer Science diploma program in Stuttgart, the alternative approach of a real inter-faculty cooperation was chosen. The new minor Scientific Computing 11, one of fifteen possible choices including Linguistics, Music Theory, Business Administration, or Mathematics, consists of a math column and 
an applications column completely provided by the mathematics department or several engineering faculties, resp. This ensures that all computer science students who want to specialize in CSE really sniff some air from mathematics and at least one field of application.

Instead of implementing specializations within existing programs, the creation of new study programs is the other possible strategy. In the majority of cases, at least at the moment, these new programs are mere graduate ones, typically master programs. As before, the underlying idea is that CSE knowledge is regarded as an extension of a conventional engineering education, e.g., giving to it some computerization and, thus, some modern flavour. Hence, the general assumption here is that all candidates already have some first relevant degree, typically a bachelor degree or a diploma degree from one of the German universities of applied science (Fachhochschulen). Next, the Rechnergestützte Wissenschaften program [1] of the ETH Zürich (hitherto a diploma program, now also a bachelor program, and in the future a bachelor-master combination) is some kind of a hybrid type. Although being a complete diploma program, the specific education starts in the third year only, since the first two years are identical to either mathematics, physics, chemistry, computer science, or some engineering subject. Finally, an example of a completely newly designed CSE program is the already mentioned Computational Engineering [6] in Erlangen, consisting of both a bachelor and a master component. This is, of course, the most consequent implementation of CSE education, which also offers most chances of a curriculum really tailored to the interdisciplinary needs of numerical simulation.

It is not clear which is the best strategy to combine a high-quality education really required by both the industrial and the academic job market with an economic usage of university resources. However, it is quite obvious that doing numerical simulations on a high level requires knowledge, skills, and experience in parts of mathematics, computer science, and in the respective field of application. And it, therefore, must be doubted whether this objective can be reached with just one of the "feel-the-flavour-type" programs within one discipline.

\section{National/German or International/English?}

The program language is another difference we discover when comparing the various CSE-related programs in Germany. Since one big motivation for master programs was to attract international students to our universities, most of them were designed as international programs with all or, at least, the vast majority of the courses being offered in English language. CSE, COMMAS, and COME are typical representatives of this development. The strategic drawback is that, at least at present, this approach keeps most of the German students out of these programs. $C E$ in Erlangen is the hybrid model, offering most of the bachelor program in German (in order to integrate German students into the CSE education), but then offering the master basically in English language (in order to integrate foreign students into the CSE education). And, finally, the Swiss Rechnergestützte Wissenschaften program is completely given in German. This is definitely one of the not that frequent examples of a CSE study program that has been completely designed and implemented for the home students. 
The language issue goes beyond this paper's scope. If, after the bachelormaster system, also the English language will gain general acceptance at German universities, it will be answered for CSE, anyway. On the other hand, if we continue distinguishing between national and international study programs, we shall see both German-language and English-language programs in the future.

\section{Target Groups and Applicants}

Of course, the language question is highly related to the question of whom a CSE program wants to address, primarily. But this target group issue is not restricted to countries or languages, there is also a disciplinary aspect. The two computational mechanics programs COME and COMMAS considered here address a quite narrow group of students - basically those with a bachelor degree in some mechanics-related field such as civil or mechanical engineering. This strategy allows to start immediately with special courses and to reach an impressive depth of studies within the three semesters. Nevertheless, the lecturers complain the obvious heterogeneity of the students (which is, here, probably more due to different previous levels of education than due to disciplines).

In contrast to this, the CSE program is open to a broad spectrum of degrees, since its regulations just require a bachelor degree in a related field including civil, mechanical, chemical, or electrical engineering, physics, chemistry, but also computer or aerospace engineering. Thus, the knowledge in some specific field of application may differ a lot among the participants of the program. However, the basic knowledge concerning advanced mathematics and basic programming skills is more depending on countries and universities than on disciplines. Hence, the umbrella character of CSE can handle this, providing a sound education in mathematical and informatical fundamentals of scientific computing and assigning the subject of application to each student according to his or her background. This way, mechanical engineers do computational fluid mechanics or computational structural mechanics, whereas physicists specialize in computational physics, e.g.

Especially for international target groups, one of the most urgent problems is applicant selection. For CSE, the situation is probably even harder due to its interdisciplinary character, which entails that there will always be candidates with different degrees. At present, the are a lot of discussions concerning reasonable strategies for making the process of applicant selection both faster and cheaper, resp., in order to avoid preparation semesters which are expensive both for students and universities, and to improve the hit rate. For that, the existing mechanisms of entrance exams or phone interviews etc. will have to be augmented by blended learning approaches combining online self-study components with special compact courses immediately before the first semester starts.

\section{Concluding Remarks}

In this paper, some topical issues concerning CSE programs in Germany were discussed. With several examples of relevant programs, the questions of the focus 
and structure of a CSE program, of different ways to implement such programs, and the question of target groups concerning both nationalities and disciplines were addressed. Unfortunately, the opinion that CSE is nothing but a private subdiscipline that has recently emerged and that can be completely dealt with internally is rather widespread throughout many faculties - even if most of those responsible will always emphasize its interdisciplinary character in public. This, to some extent, often has strange and even negative consequences for the design of CSE specializations or CSE programs. There are even cases where a so-called CSE specialization is more a misleading package than something really deserving its name. However, for integrated specializations as well as for independent programs, involving all contributing disciplines - here, first of all, mathematics, computer science, and the various fields of applications have to be mentioned is an essential precondition of a CSE education that is successful in the longer term and that really fulfils the needs of CSE as a discipline.

\section{References}

1. ETH Zürich. Rechnergestützte Wissenschaften - diploma, bachelor, and master programs. www.rw.ethz.ch/.

2. Freie Universität Berlin. Scientific Computing - international master program. www.math.fu-berlin.de/teach/webstud/studium/msc.htm.

3. Technische Universität München. Computational Mechanics (COME) - international master program. www.come.tum.de/.

4. Technische Universität München. Computational Science and Engineering - international master program. www.cse.tum.de/.

5. Technische Universität München, Universität Erlangen-Nürnberg, Universität Stuttgart. Ferienakademie. www5.in.tum.de/FA/.

6. Universität Erlangen-Nürnberg. Computational Engineering - bachelor and international master programs. www10.informatik.uni-erlangen.de/CE/.

7. Universität Stuttgart. Computational Mechanics of Material and Structures (COMMAS) - international master program. www.msc.commas.uni-stuttgart.de/.

8. Universität Stuttgart. Computational Physics - bachelor program. www.physik.uni-stuttgart.de/studium/bachelor/.

9. Universität Stuttgart. Computational Science and Engineering - specialization within the diploma program Mechanical Engineering. www.uni-stuttgart.de/mawesen/studienrichtungen.html/.

10. Universität Stuttgart. Modelling and Simulation - specialization within the diploma program Civil Engineering. www.iwb.uni-stuttgart.de/stuko/index.html.

11. Universität Stuttgart. Scientific Computing - minor within the diploma program Computer Science. www.informatik.uni-stuttgart.de/fakultaet/lehre/informatik/. 